\title{
Coupled rotational motion of Mercury
}

\author{
N. Rambaux ${ }^{1,2}$, A. Lemaitre ${ }^{1}$, and S. D’Hoedt ${ }^{1}$ \\ 1 Depart. of Mathematics, Facultés Univ. N.D. de la Paix, 8 Rempart de la Vierge, 5000 Namur, Belgium \\ e-mail: Nicolas.Rambaux@fundp.ac.be \\ 2 Royal Observatory of Belgium, 3 Avenue Circulaire, 1180 Brussels, Belgium
}

Received 30 October 2006 / Accepted 2 March 2007

\section{ABSTRACT}

\begin{abstract}
We present a simple dynamical model of the rotation of Mercury in which the Hermean rotation is composed of two commensurabilities: (i) a 3:2 spin-orbit resonance between fast variables and (ii) a 1:1 synchronous precession of both orbital and rotational nodes. We investigate the coupling between these two degrees of freedom. First, we study the global phase space of Mercury and quantify the libration areas. Second, we concentrate on the present location of Mercury. The impact of the slow degree of freedom on the fast one can be modeled through the adiabatic invariant, whereas the impact of the fast degree of freedom on the slow one is clearly represented by Poincaré sections. In addition, the adiabatic invariant theory leads to a simple analytical model of the rotation of Mercury where the two coupled degrees of freedom are taken into account. This model can be used in different applications that require a first-order rotational motion such as the one describing the influence of the precession and rotation of the planet on the orbit of an artificial satellite around Mercury.
\end{abstract}

Key words. celestial mechanics - planets and satellites: individual: Mercury - methods: analytical - methods: numerical

\section{Introduction}

Radar observations have revealed that Mercury's rotational period is 58.6 days (Pettengil \& Dyce 1965) rather than the previously accepted value of 88 days. Colombo (1965) pointed out that the ratio of the orbital to rotational period is exactly $3: 2$, a unique case of the asynchronous spin-orbit resonance of our Solar System. In the peculiar case of Mercury, Celletti \& Falconi (1992) and Celletti \& Chierchia (2000) investigated the stability of spin-orbit resonances by using the KAM theory successfully. Colombo (1965), Peale (1969), and Beletski (1972) studied the dynamical motion of Mercury by the generalized Cassini laws. Nevertheless, the rotational motion of Mercury has been understudied compared to the synchronous spin-orbit resonance cases (see for example Bouquillon et al. 2003).

Two commensurabilities characterize the rotational motion of Mercury: (i) a 3:2 spin-orbit resonance between two fast variables (the mean longitude and the spin rotation angle of Mercury) and (ii) a 1:1 synchronous precession of the two nodes (the orbital and rotational ones). These motions are wholly forced by the point source Sun acting on Mercury and generating two modes of libration. Rambaux \& Bois (2004) determine the two proper or resonant frequencies at 15.847 and 1066.91 years by using the SONYR model. SONYR is the acronym of Spin-Orbit $N$-body Relativistic model, and it was used to perform a very accurate rotational motion of Mercury included in the Solar System. More recently, D'Hoedt \& Lemaitre (2004a) developed a Hamiltonian approach to the rigid rotational motion of Mercury, giving a first approximation of the rotation composed of two degrees of freedom (Lemaitre et al. 2006). The reference model is truncated, averaged, and simplified; several canonical transformations in chain are used to uncouple these two main degrees of freedom. Despite these several steps, the two corresponding proper frequencies, obtained by this analytical approach (15.857 and 1065.08 years) are very close to those obtained by Rambaux \& Bois (2004) by using the SONYR model. This means that the analytical model could be considered as a good first approximation of the resonant rotation of Mercury. It is relevant to go into the details in its structure, to understand how the coupling between the two degrees of freedom acts and how each motion interferes in the motion of the other degree of freedom.

The objective of this paper is to study the coupled simplified dynamics of Mercury, first in the global two-dimensional phase space and second, locally in the neighborhood of the present resonant position of Mercury.

In the next section after a few statements and basic formulae, we determine the equilibrium points (corresponding to the socalled Cassini equilibria), plot the phase space, and quantify the amplitudes of the libration zones. In the third section, we focus on the present position of Mercury and investigate the mutual interactions and deformations induced by the motion on each degree of freedom. We show that the impact of the slow degree of freedom on the fast one can be modeled through the adiabatic invariant theory and that the impact of the fast degree of freedom on the slow one is clearly represented by Poincare sections.

\section{The two-dimensional Hamiltonian approach}

To describe the phase space corresponding to the rotational motion of Mercury, we start with the Hamiltonian developed by D’Hoedt \& Lemaitre (2004a,b).

\subsection{The variables}

The orbit of Mercury is described by the classical Delaunay's elements: $L_{\mathrm{o}}, G_{\mathrm{o}}, H_{\mathrm{o}}, l_{\mathrm{o}}, g_{\mathrm{o}}, h_{\mathrm{o}}$. The angle $l_{\mathrm{o}}$ is the mean anomaly, $g_{\mathrm{o}}=\omega_{\mathrm{o}}$ is the argument of pericenter, and $h_{\mathrm{o}}=\Omega_{\mathrm{o}}$ the longitude of the ascending node, measured on some suitable inertial 
reference plane. The inertial reference system can be linked to the ecliptic plane fixed at some epoch or to a suitable Laplace plane fixed at some epoch (Lemaitre et al. 2006). The capital letters designate the associated conjugated momenta and are defined by

$$
\begin{aligned}
& L_{\mathrm{o}}=m \sqrt{\mu a} \\
& G_{\mathrm{o}}=L_{\mathrm{o}} \sqrt{1-e^{2}} \\
& H_{\mathrm{o}}=G_{\mathrm{o}} \cos i_{\mathrm{o}}
\end{aligned}
$$

where $e$ is the eccentricity, $a$ the semimajor axis, and $\mu$ is equal to $\mathcal{G}(m+M)$, where $\mathcal{G}$ is the universal constant of gravitation, $m$ and $M$ are the masses of Mercury and the Sun, respectively.

To describe the rotational motion of a rigid body around its center of mass, we use Andoyer's variables (Deprit 1967). They consist of two linked sets of Euler's angles: the first set $(h, K,-)$ locates the position of the frame linked to the rotational angular momentum with respect to the inertial frame, and the second set $(g, J, \ell)$ locates the body frame (corresponding to the principal axes of inertia) in the previous frame tied to the angular momentum. There, $L=G \cos J, G$ and $H=G \cos K$ are the conjugate momenta of the angles $(\ell, g, h)$ where $G$ is the norm of the angular momentum and $J$ the angle between the angular momentum and the third axis of inertia. The variable $K$ is called the inertial obliquity (the obliquity is measured with respect to the inertial reference system). Andoyer's variables present two virtual singularities when $K$ and $J$ are equal to zero. However, the sum of the three angular variables $\ell, g$, and $h$ is always well-defined. In this model, we consider that $J=0$ (a three-dimensional approach can be found in D'Hoedt \& Lemaitre 2004b) and we use the following modified Andoyer's variables

$$
\begin{array}{ll}
\lambda_{1}=\ell+g+h & \Lambda_{1}=G \\
\lambda_{3}=-h & \Lambda_{3}=G-H=G(1-\cos K)
\end{array}
$$

in which we keep the indices 1 and 3 as in the reference paper (D'Hoedt \& Lemaitre 2004a).

The rotation period of Mercury is nearly equal to $2 / 3$ of the orbital period. Also, as studied by Peale (1969) and Beletski (1972), the node of the orbit and the node of the equator have, on average, the same period. Consequently, we defined two resonant angles:

$$
\begin{aligned}
& \sigma_{1}=\lambda_{1}-\frac{3}{2} l_{\mathrm{o}}-h_{\mathrm{o}}-g_{\mathrm{o}} \\
& \sigma_{3}=\lambda_{3}-h_{\mathrm{o}} .
\end{aligned}
$$

The two sets of canonical variables (orbital and rotational) are then mixed in these commensurabilities. In order to keep a canonical transformation, we must associate to $l_{\mathrm{o}}$ a new conjugated momentum:

$\Lambda_{\mathrm{o}}=L_{\mathrm{o}}+\frac{3}{2} \Lambda_{1}$,

where $\Lambda_{1}$ is, in this set, the conjugated momentum of $\sigma_{1}$.

\subsection{The Hamiltonian}

The Hamiltonian (5) is composed of four terms: the first one corresponds to the Keplerian problem, the second one is due to the precession of the orbital plane. It is the only contribution of the planetary perturbations, given by a constant precession term $\mu_{1}$. It plays an important role in the stability of the equilibria as shown in D'Hoedt et al. (2006). The third term in the Hamiltonian is related to the free rotation of a rigid body about its center of mass, and the fourth one is due to the solar torque acting on Mercury developed in spherical harmonics of degree 2.
The development of the Hamiltonian is truncated in eccentricity and inclination, limited to the second degree for the harmonics, averaged over the short periods, which gives the following expression:

$$
\begin{aligned}
\langle\mathcal{H}\rangle= & -\frac{m^{3} \mu^{2}}{2\left(\Lambda_{0}-\frac{3 \Lambda_{1}}{2}\right)^{2}}-\mu_{1}\left(H_{\mathrm{o}}+\Lambda_{1}-\Lambda_{3}\right) \\
& +\frac{\Lambda_{1}^{2}}{2 I_{3}}-\mathcal{F}\left(C_{2}^{0}(e) \sum_{i=0}^{2} a_{0 i} \cos \left(i \sigma_{3}\right)\right. \\
& \left.+C_{2}^{2}(e) \sum_{i=0}^{4} a_{2 i} \cos \left(2 \sigma_{1}+i \sigma_{3}\right)\right)
\end{aligned}
$$

with

$C_{2}^{0}(e)=C_{2}^{0}\left(1+\frac{3}{2} e^{2}\right), \quad C_{2}^{2}(e)=6 C_{2}^{2}\left(\frac{7}{2} e-\frac{123}{16} e^{3}\right)$

and

$\mathcal{F}=\frac{G M m^{7} \mu^{3} R_{e}^{2}}{2\left(\Lambda_{0}-\frac{3 \Lambda_{1}}{2}\right)^{6}}$

where $a_{0 i}$ and $a_{2 i}$ depend on $i_{\mathrm{o}}$ and $K, R_{\mathrm{e}}$ is the equatorial radius of Mercury, $I_{3}$ is the third principal moment of inertia, $C_{2}^{0}$, and $C_{2}^{2}$ are the spherical harmonics of Mercury.

\subsection{Cassini's equilibria}

The equilibrium points (also called Cassini's equilibria) are obtained by equating the right hand sides of the canonical equations of motion to zero:

$\frac{\mathrm{d} \sigma_{k}}{\mathrm{~d} t}=\frac{\partial\langle\mathcal{H}\rangle}{\partial \Lambda_{k}}=0 ; \quad \frac{\mathrm{d} \Lambda_{k}}{\mathrm{~d} t}=-\frac{\partial\langle\mathcal{H}\rangle}{\partial \sigma_{k}}=0$

for $k=1$ and $k=3$.

These equations admit equilibria defined by the doublet $\left(\sigma_{1}, \sigma_{3}\right)$ equal to $(0,0),(0,360),(180,0),(180,360)$ given in degrees, denoted hereafter deg, and the conditions:

$\frac{\partial\langle\mathcal{H}\rangle}{\partial \Lambda_{1}}=0 ; \quad \frac{\partial\langle\mathcal{H}\rangle}{\partial \cos K}=0$.

Let us note that $\sigma_{1}=0 \mathrm{deg}$ (respectively $\sigma_{1}=180 \mathrm{deg}$ ) means that the axis of smallest (respectively intermediate) inertia points toward the Sun at each perihelion passage and that $\sigma_{3}=0 \mathrm{deg}$ represents the alignment of the lines of node of the orbit and of the equator. On the contrary $\sigma_{3}=360 \mathrm{deg}$ expresses the antialignment of the two nodes.

Equations (7) give the following transcendental equation

$$
\begin{aligned}
\frac{\mathcal{F}}{I_{3} \mu_{1}}\left(C_{2}^{0}(e) F_{1}+C_{2}^{2}(e) F_{2}\right) & =-\frac{3 m^{3} \mu^{2}}{2 L_{0}^{3}} \\
- & \frac{9 \mathcal{F}}{L_{0}}\left(C_{2}^{0}(e) F_{3}+C_{2}^{2}(e) F_{4}\right)-\mu_{1} \cos K,
\end{aligned}
$$

where the functions $F_{1}, F_{2}, F_{3}$ and $F_{4}$ are defined by:

$$
\begin{aligned}
& F_{1}=\frac{3}{2} \sin \left(2 i_{\mathrm{o}}-2 K\right) / \sin K \\
& F_{2}=\left[-\frac{1}{2} \sin \left(i_{\mathrm{o}}-K\right)-\frac{1}{4} \sin \left(2 i_{\mathrm{o}}-2 K\right)\right] / \sin K \\
& F_{3}=-\frac{1}{4}-\frac{3}{4} \cos \left(2 i_{\mathrm{o}}-2 K\right) \\
& F_{4}=\frac{3}{8}+\frac{1}{2} \cos \left(i_{\mathrm{o}}-K\right)+\frac{1}{8} \cos \left(2 i_{\mathrm{o}}-2 K\right)
\end{aligned}
$$


Table 1. The numerical values of the parameters. (a) From D'Hoedt et al. (2006) and (b) from Anderson et al. (1987).

\begin{tabular}{llll}
\hline \hline Name & Quantity & Value & Unity \\
\hline$a$ & Semi-major axis (a) & $57.9 \times 10^{6}$ & $\mathrm{~km}$ \\
$e$ & Eccentricity (a) & 0.206 & \\
$i_{0}$ & Inclination of the orbital plane (a) & 7 & $\mathrm{deg}$ \\
$R_{\mathrm{e}}$ & Equatorial radius of Mercury & 2440 & $\mathrm{~km}$ \\
$C_{2}^{0}$ & Spherical harmonics (b) & $60.0 \times 10^{-6}$ & \\
$C_{2}^{2}$ & Spherical harmonics (b) & $10.0 \times 10^{-6}$ & \\
$I_{3}$ & polar moment of inertia (a) & 0.34 & \\
$\mu_{1}$ & Precessional constant (a) & $0.2244 \times 10^{-4}$ & $\mathrm{rad} \mathrm{y}^{-1}$ \\
\hline
\end{tabular}

Table 2. Values of the equilibria ecliptic obliquity from the analytical and numerical studies in the case $\left(\sigma_{1}, \sigma_{3}\right)=(0,0)$ deg.

\begin{tabular}{crr}
\hline \hline Equilibria ecliptic obliquity & Analytically (deg) & Numerically (deg) \\
\hline$K_{1}$ & 7.027 & 7.028 \\
$K_{2}$ & 102.098 & 102.101 \\
$K_{3}$ & 186.968 & 186.969 \\
$K_{4}$ & 271.908 & 271.909 \\
\hline
\end{tabular}

There are four real solutions to this equation for each doublet $\left(\sigma_{1}, \sigma_{3}\right)$ depending on the values of the dynamical and geophysical parameters (the values are listed in Table 1). We choose the ecliptic reference frame of the standard epoch J2000 as the inertial one. For the doublet $(0,0)$, we compute the value of $K^{\star}$, the equilibrium value of the ecliptic obliquity, first from the analytical equation (8) and second from the numerical integration, see Table 2 . The case $\mu_{1}=0$ would give $K_{1}=i_{0}=7 \mathrm{deg}$. Consequently, the increase of about $0.027 \mathrm{deg}$ for $K_{1}$ in Table 2 comes from the precession (D'Hoedt et al. 2006).

The existence of these solutions ( 2 or 4 equilibria) has already been pointed out by several authors (Colombo 1965; Peale 1969, 1974; Beletskii 1972). However, our contribution has the advantage of calculating these equilibrium positions with recent values of the gravitational coefficients (Anderson et al. 1987), which introduce slight quantitative differences in the numerical results.

\subsection{The global phase space $\left(\sigma_{3}, K\right)$}

We represent the dynamics of the Hamiltonian in the plane $\left(\sigma_{3}, K\right)$ describing the phase space of the second degree of freedom. The four Hamiltonian equations of motion are numerically integrated for fixed initial conditions $\left(\sigma_{1}=\sigma_{10}=0 \mathrm{deg}\right.$, $\Lambda_{1}=\Lambda_{10}=13.303 \mathrm{~m} R_{\mathrm{e}}^{2} / \mathrm{yr}$, where the equatorial radius of Mercury $R_{\mathrm{e}}$, its mass $m$, and the terrestrial year are the units of length, mass, and time, respectively) and by assuming that the orbit of Mercury precesses at a constant rate. The behavior of the obliquity is plotted in Fig. 1 for $K<180 \mathrm{deg}$ and in Fig. 2 for $180<K<360 \mathrm{deg}$. We find good agreement with the eight fixed points of the analytical computation (see Table 2). Four of the equilibria are located on the vertical line $\sigma_{3}=0$ and the others on the vertical line $\sigma_{3}=180 \mathrm{deg}$. Six points are centers and two are saddle points characterizing the stable and unstable behaviors of the dynamics around these fixed points.

In addition, Figs. 1 and 2 give the amplitudes of the stable zones, the so-called "cat eyes" characterizing the pendulum centers. For the present location of Mercury $\left(K_{1}\right)$, the ecliptic obliquity has to be smaller than $14 \mathrm{deg}$ to capture Mercury in $\sigma_{3}$ resonance. The width of the area surrounding the equilibrium $K_{3}$ is around $12 \mathrm{deg}$ and around $5 \mathrm{deg}$ for $K_{4}$.

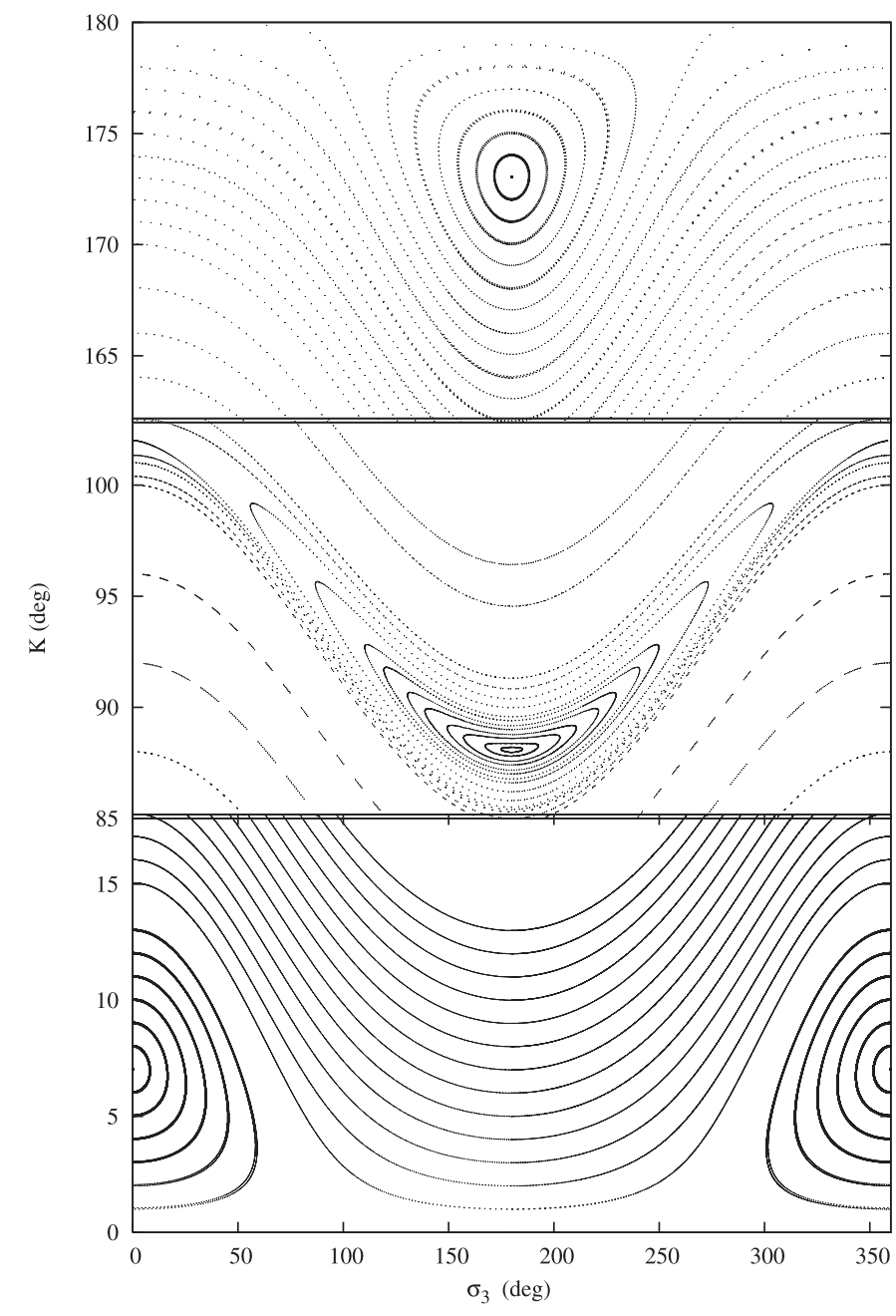

Fig. 1. $\left(\sigma_{3}, K\right)$ phase space. Each panel focuses on an equilibrium point over an interval of $18 \mathrm{deg}$ for values of $K$ lower than $180 \mathrm{deg}$. The area surrounding the equilibrium point located at $(0,7)$ deg is expected to contain the actual position of the spin axis of Mercury.

\subsection{The global phase space $\left(\sigma_{1}, \Lambda_{1}\right)$}

We show the dynamics of the Hamiltonian in the plane $\left(\sigma_{1}, \Lambda_{1}\right)$ corresponding to the phase space of the first degree of freedom. We fixed initial conditions of the second degree of freedom $\left(\sigma_{3}=\sigma_{30}=0, \Lambda_{3}=\Lambda_{30}=0.09917 \mathrm{~m} R_{\mathrm{e}}^{2} / \mathrm{yr}\right)$ and assumed, as previously, that the orbit of Mercury precesses at a constant rate.

Figure 3 of the phase space $\left(\sigma_{1}, \Lambda_{1}\right)$ presents a pendulumlike behavior of the first degree of freedom around a fixed point ( $0.0 \mathrm{deg} ; 13.303 \mathrm{~m} R_{\mathrm{e}}^{2} / \mathrm{yr}$ ), which is a stable point (see also a Poincaré section plotted in Rambaux \& Bois 2004, Fig. 2). The width of the resonance area measured on the $\Lambda_{1}$ axis is around $0.26 \mathrm{~m} R_{\mathrm{e}}^{2} / \mathrm{yr}$.

\section{The local coupled resonant motion}

\subsection{Phase space}

We focus on the dynamics in the neighborhood of the first equilibrium $K=K_{1}$, where Mercury is assumed to be. Consequently, we perform a numerical integration of the Hamiltonian $\langle\mathcal{H}\rangle$ Eq. (5) to obtain a detailed local description of the two degree of freedom phase space. In order to avoid the singularity at the origin, we represent the dynamical motion in terms of 


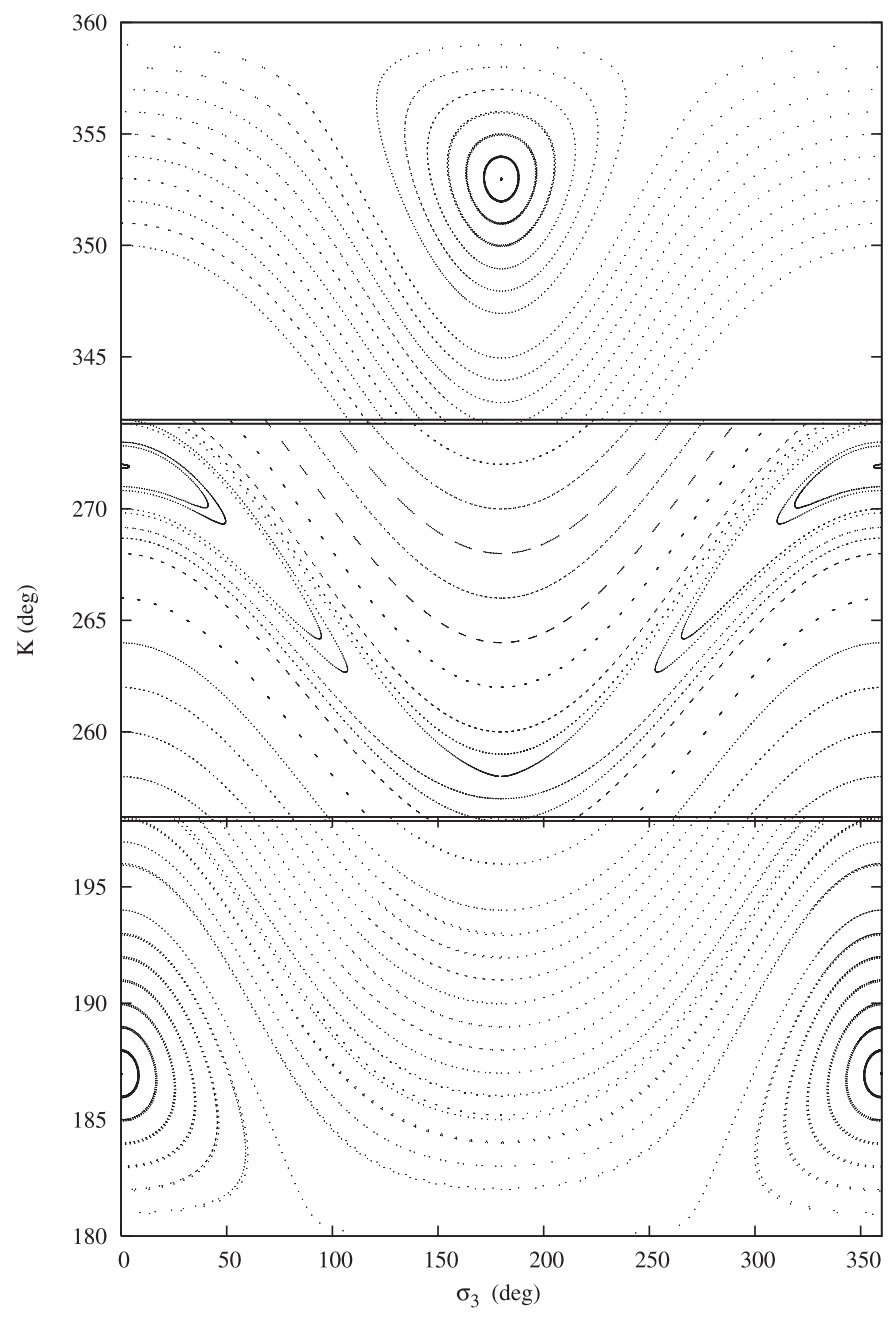

Fig. 2. $\left(\sigma_{3}, K\right)$ phase space. Each panel focuses on an equilibrium point over an inteval of $18 \mathrm{deg}$ for values of $K$ upper than $180 \mathrm{deg}$.

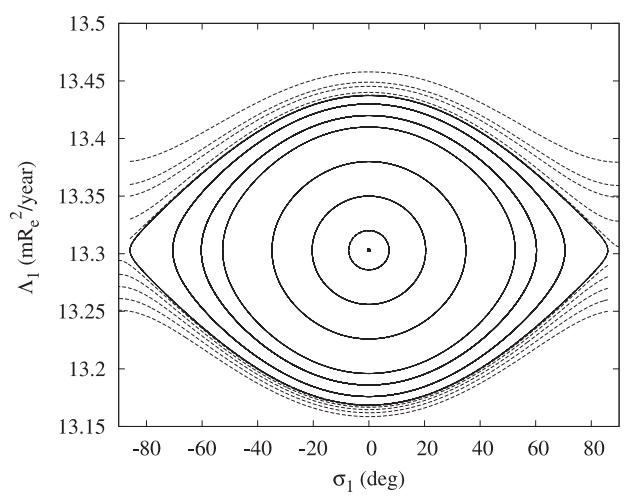

Fig. 3. Phase space $\left(\sigma_{1}, \Lambda_{1}\right)$. The curve presents a pendulum-like behavior with a librational area around $0.26 \mathrm{~m} R_{\mathrm{e}}^{2} / \mathrm{yr}$ on the $\Lambda_{1}$ axis.

Cartesian coordinates $\left(x_{1}, y_{1}\right)$ and $\left(x_{3}, y_{3}\right)$ by the canonical transformations:

$\left\{\begin{array}{l}x_{i}=\sqrt{2 \Lambda_{i}} \cos \sigma_{i} \\ y_{i}=\sqrt{2 \Lambda_{i}} \sin \sigma_{i}\end{array}\right.$

for $i=1$ or 3 . In addition, we normalize $\Lambda_{i}$ to $\mathrm{m} R_{\mathrm{e}}^{2} /$ year and all the following variables are adimensional.

Figure 4 shows the rotation of Mercury in the planes $\left(x_{1}, y_{1}\right)$ and $\left(x_{3}, y_{3}\right)$ for the initial conditions $x_{10}=5.1581, y_{10}=0.00, y_{30}=0.00$ and for $x_{30}$ chosen between 0.0637 and 0.5079 . The curves of the same color, Figs. $4 \mathrm{a}$ and $4 \mathrm{~b}$ correspond to the same initial conditions. The curves in the plane $\left(x_{1}, y_{1}\right)$ show a typical behavior of first-order resonance in the resonance area (also called libration area), and the phase plane $\left(x_{3}, y_{3}\right)$ corresponds to a pendulum-like behavior. The first degree of freedom $\left(x_{1}, y_{1}\right)$ is clearly associated to the first proper period of 15 years and the second one $\left(x_{3}, y_{3}\right)$ to the second proper period of 1066 years. However, the mutual influence of both periods is obvious on both planes ; and due to the fact that the periods are very distinct (a ratio around 70), the analysis of the perturbation that they induce on each other, requires specific tools. For Sects. 3.2 and 3.3, we focus on the red curves of Figs. $4 \mathrm{a}$ and $\mathrm{b}$ corresponding to $y_{30}=0.0637$, i.e. showing a strong interaction between the two degrees of freedom. For the Sect. 3.4, we investigate the Poincaré section for the blue curve, i.e. $y_{30}=0.4453$.

\subsection{Slice cutting}

To illustrate the motion in $\left(x_{1}, y_{1}\right)$ plane, we adapt an enlightening method developed by Froeschle (1972) to represent the four-dimension map: slice cutting. We consider the space of the points $P=\left(x_{1}, y_{1}, t\right)$ for $t=[0,1053]$ years. We define one slice as the point family $\left(x_{1 k}, y_{1 k}\right)$ of projected points $P$ on $(x y)$ plane such that

$\left\{(x, y, t): t_{k-1}<t \leqslant t_{k}\right\}$

where $t_{k}$ is equal to $k h$. According to the proper periods and initial conditions (close to the equilibrium) of our problem, we fix $h=17$ years, and $k$ varies from 0 to 62 (so the time interval is 1053 years). We plot each slice, the $\left(x_{1 k}, y_{1 k}\right)$ plane corresponding to a value of $k$, side by side, to give a better idea of the surface and especially to study the sheets in detail. We present a sample of slice taken at various dates (quoted on the slice) in order to illustrate the behavior of the dynamical interactions (the complete sequence contains 62 slices). The complete display of the motion allows identification of the different dynamical phases occuring during the spin-orbit motion. The slices shown in Fig. 5 have to be seen from the left to right and from top to bottom.

The section on each slice evolves with time due to the interaction with the second degree of freedom $\left(x_{3}, y_{3}\right)$. We start from a banana shape, and the section is deformed along the upper branch before coming back again to the banana shape. At this point, the section is going to elongate according to the lower branch and finally return of its initial form. The period of time for this pulsation is the second proper frequency.

From these figures, we deduce two particular features of the behavior: (i) the area enclosed by the orbit seems to be constant and (ii) the center of the orbit undergoes a regular oscillation during one period.

\subsection{Adiabatic behavior}

The geometrical area enclosed by the orbit is $2 \pi \mathcal{J}$, where $\mathcal{J}$ is the action variable defined as (Henrard 2005):

$\mathcal{J}=\frac{1}{4 \pi} \oint x_{1} \mathrm{~d} y_{1}-y_{1} \mathrm{~d} x_{1}$

We can evaluate $\mathcal{J}$ at each step of our numerical integration, thanks to the above expression. Figure 6 shows that the action $\mathcal{J}$ is almost constant over 3000 years, proving that the second degree of freedom acts as a very slow parameter on the first degree, in an adiabatic way. Let us demonstrate this feature analytically. 

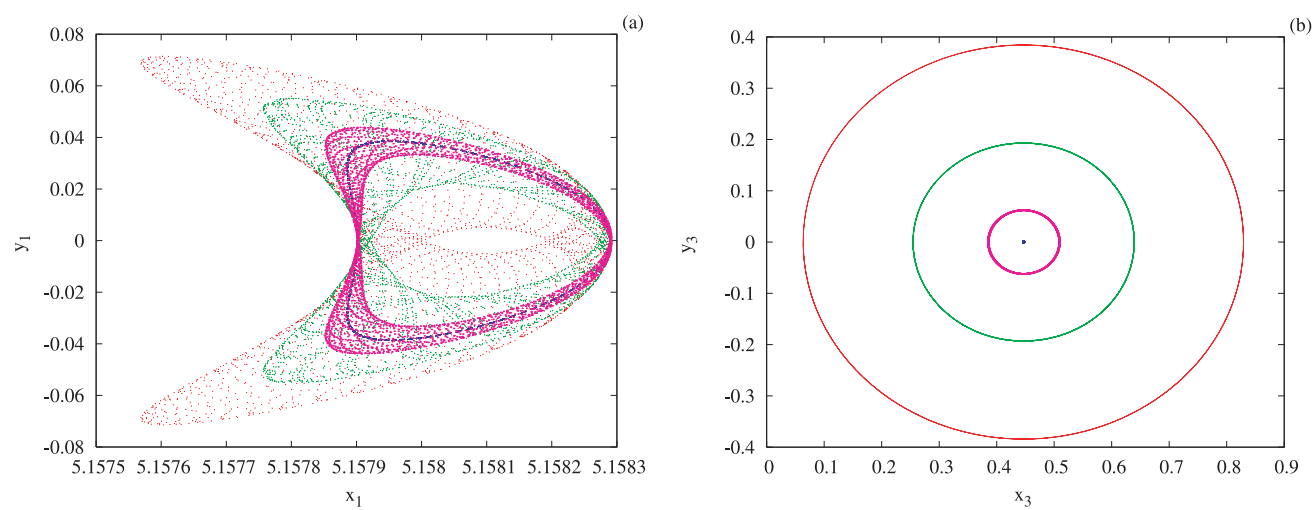

Fig. 4. a) Phase plane $\left(x_{1}, y_{1}\right)$ and b) phase plane $\left(x_{3}, y_{3}\right)$. The curves of the same color correspond to the same initial conditions. The blue curves are the closest to the equilibrium position.
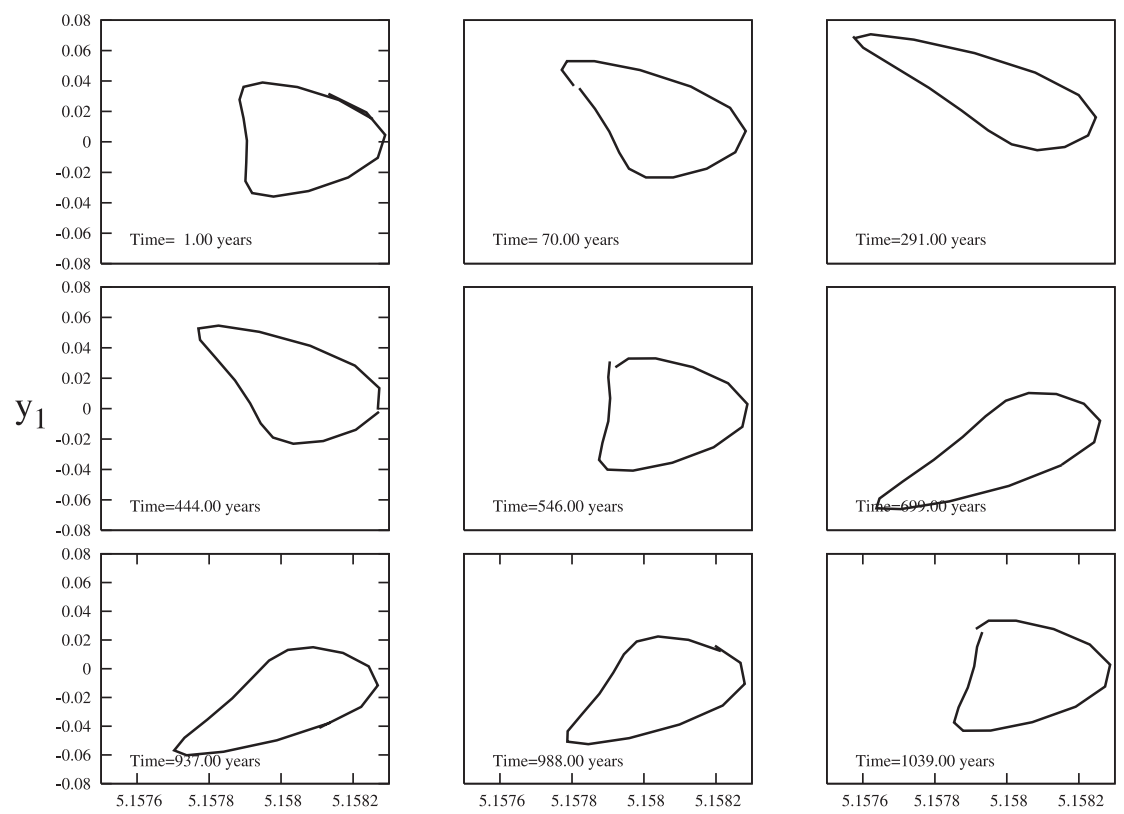

$\mathrm{x}_{1}$

Fig. 5. Behavior of the orbit in the $\left(x_{1}, y_{1}\right)$ plane over 1053 years by slices of 17 years taken at various times.

Locally, we can expand the Hamiltonian in powers of $\xi_{1}, y_{1}$, $\xi_{3}$, and $y_{3}$, where $\xi_{1}$ and $\xi_{3}$ are obtained by a translation of $x_{1}$ and $x_{3}$ at the equilibrium corresponding to $K_{1}$ :

$\xi_{1}=x_{1}-x_{1, K_{1}} \quad$ with $\quad x_{1, K_{1}}=5.158291$

$\xi_{3}=x_{3}-x_{3, K_{1}} \quad$ with $\quad x_{3, K_{1}}=0.445344$.

Let us now concentrate on the behavior of the system in the $\left(\xi_{1}, y_{1}\right)$ plane. As a first approximation, we obtain a quadratic form with the following terms:

$\mathcal{K}_{2}=a \xi_{1}^{2}+2 c \xi_{1} \xi_{3}+e y_{1}^{2}+2 g y_{1} y_{3}$

where $a=39.12645,2 c=-0.00051, e=0.00100,2 g=$ 0.00017 (D'Hoedt \& Lemaître 2004). Where $\xi_{3}$ and $y_{3}$ are considered as slow explicit functions of time,

$\xi_{3}=A_{3} \cos \theta$

$y_{3}=B_{3} \sin \theta$.

Here, $\dot{\theta}=\omega_{3}$ is the (slow) proper frequency corresponding to the equilibrium, and $A_{3}$ and $B_{3}$ are fixed (small) amplitudes. In the Hamiltonian $\mathcal{K}_{2}$, the precession constant $\mu_{1}$ is negligible over the time scale of this study (about one thousand years). In a twodimensional canonical formalism, the Hamiltonian becomes

$$
\mathcal{K}=\mathcal{K}_{2}\left(\xi_{1}, y_{1}, \theta\right)+\omega_{3} \Theta
$$

with $\Theta$ the conjugated momentum to $\theta$.

We perform a canonical transformation (a parameterdependent translation to center the ellipse and the corresponding correction on the other degree of freedom):

$$
\begin{aligned}
\xi_{1}^{t}=\xi_{1}+\frac{c}{a} A_{3} \cos \theta & \theta^{t}=\theta \\
y_{1}^{t}=y_{1}+\frac{g}{e} B_{3} \sin \theta & \Theta^{t}=\Theta-R\left(y_{1}, \xi_{1}, \theta\right) .
\end{aligned}
$$

By using the canonical conditions $\left[\Theta^{t} ; y_{1}^{t}\right]=0$ and $\left[\Theta^{t} ; \xi_{1}^{t}\right]=0$, where [; ] stands for Poisson brackets, we obtain the following explicit expression for the remainder function $R$ :

$R\left(y_{1}, \xi_{1}, \theta\right)=\frac{c}{a} A_{3} \sin \theta y_{1}+\frac{g}{e} B_{3} \cos \theta \xi_{1}$.

The Hamiltonian can be written as

$\mathcal{K}=a \xi_{1}^{t^{2}}+e y_{1}^{t^{2}}+\omega_{3}\left(\Theta^{t}+R\left(y_{1}^{t}, \xi_{1}^{t}, \theta^{t}\right)\right)+\ldots$ 


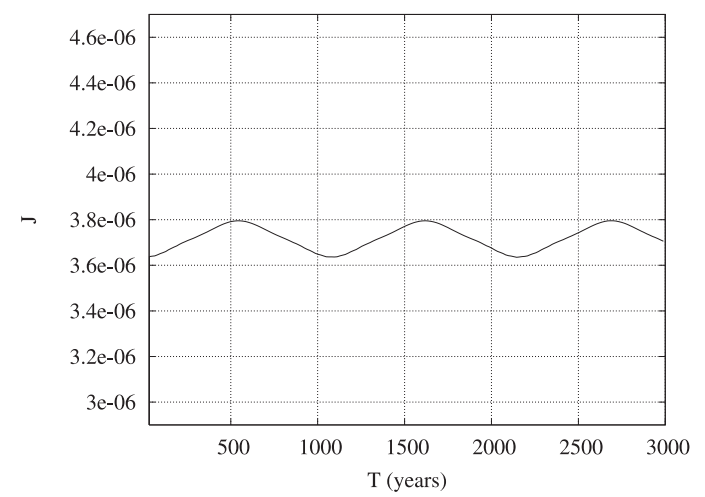

Fig. 6. Temporal evolution of the action $\mathcal{J}$ quasi-constant over 3000 years.

Using action-angle variables $J_{1}$ and $\Psi_{1}$ instead of $\xi_{1}^{t}$ and $y_{1}^{t}$, we can rewrite the Hamiltonian $K$ as

$\mathcal{K}=2 \sqrt{a e} J_{1}+\omega_{3} \Theta^{t}+\omega_{3} R\left(J_{1}, \Psi_{1}, \theta^{t}\right)+\ldots$

After a second averaging over $\Psi_{1}$, the resulting action $\bar{J}_{1}$ is a constant, related to $J_{1}$ by the relation

$J_{1}=\bar{J}_{1}+\delta J_{1}$.

Choosing $A_{3}=B_{3}=0.4$ corresponding to our numerical simulation (see Fig. $4 \mathrm{~b}$ ), we prove that the area $J_{1}$ is nearly constant, and the oscillation $\delta J_{1}$ around $\bar{J}_{1}$ is bounded by

$\left|\delta J_{1}\right|<\max \left(\omega_{3} \frac{c}{a} A_{3}, \omega_{3} \frac{g}{e} B_{3}\right) M$

where $M$ is the maximum amplitude for the selected orbit. As a consequence, $\left|\delta J_{1}\right|$ is on the order of $1.2 \times 10^{-7}$ in agreement with the numerical calculation in Fig. 6.

In the plane $\left(x_{1}, y_{1}\right)$, the guiding trajectory can be considered at any time as a close curve, centered on a slowly moving center as described in Fig. 7, along with motion of the orbit over 1070 years.

Thanks to the Hamiltonian $\mathcal{K}$ Eq. (20), a second timedependent correction on the position of the center of the ellipse can be calculated, proportional to $\omega_{3}$. It corresponds to a time-dependent translation, leading to twice-translated variables, called $\xi_{1}^{t t}$ and $y_{1}^{t t}$ and given by

$$
\begin{aligned}
& \xi_{1}^{t t}=\xi_{1}^{t}+\omega_{3} B_{3} \cos \theta \frac{g}{2 e a} \\
& y_{1}^{t t}=y_{1}^{t}+\omega_{3} A_{3} \sin \theta \frac{c}{2 e a} .
\end{aligned}
$$

In terms of these variables, the simplified Hamiltonian $\mathcal{K}$ is written as

$\mathcal{K}=a\left(\xi_{1}^{t t}\right)^{2}+e\left(y_{1}^{t t}\right)^{2}+O\left(\omega_{3}^{2}\right)+\omega_{3} \Theta^{t}$.

The explicit solutions of the Hamiltonian $\mathcal{K}$ are then

$$
\begin{aligned}
& \xi_{1}^{t t}=C_{1} \cos \left(2 \sqrt{a e} t+C_{2}\right) \\
& y_{1}^{t t}=C_{1} \sqrt{\frac{a}{e}} \sin \left(2 \sqrt{a e} t+C_{2}\right),
\end{aligned}
$$

where $C_{1}$ and $C_{2}$ are two constants of integration and the fundamental period $2 \pi /(2 \sqrt{a e})$ is equal to 15.707 years.

The complete time-dependent translation is then

$$
\begin{aligned}
& \xi_{1}=\xi_{1}^{t t}-\omega_{3} B_{3} \cos \theta \frac{g}{2 e a}-\frac{c}{a} A_{3} \cos \theta \\
& y_{1}=y_{1}^{t t}-\omega_{3} A_{3} \sin \theta \frac{c}{2 e a}-\frac{g}{e} B_{3} \sin \theta .
\end{aligned}
$$

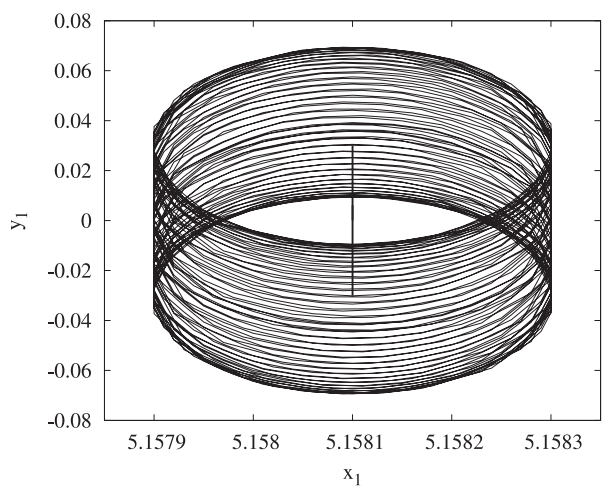

Fig. 7. The orbit in the plane $\left(x_{1}, y_{1}\right)$ in the case of the Hamiltonian $K_{2}$ and motion of the center of the guiding trajectory (straight line).

\subsection{Asymmetric shape of the curves}

Taking more significant terms in the expansion of the Hamiltonian (16) into account, we end up with the fourth-degree polynomial expression:

$$
\begin{aligned}
\mathcal{K}_{4}= & a \xi_{1}^{2}+b \xi_{1}^{3}+p \xi_{1}^{4}+2 c \xi_{1} \xi_{3}+q \xi_{1} \xi_{3}^{2}+e y_{1}^{t^{2}}+b \xi_{1} y_{1}^{2} \\
& +p y_{1}^{4}+2 g y_{1} y_{3}+q \xi_{1} y_{3}^{2}
\end{aligned}
$$

where $a, c, e$, and $g$ are already defined and $b=7.58542, q=$ -0.00115 , and $p=0.36765$. The result is an equation of motion of the fourth order with two real and two imaginary solutions.

The same procedure can be applied; however, the resulting curve is not an ellipse anymore but a more complicated curve with a banana shape, slowly moving and deforming with time. It is not symmetrical with respect to the center of the ellipse. By reproducing this truncated expression, replacing $\xi_{3}$ and $y_{3}$ by their simple time approximation, we get the following shape shown in Fig. 8 (in $\left(x_{1}, y_{1}\right)$ plane), very close to our numerical integration.

\subsection{Motion of the slow degree of freedom}

We investigate the motion of the second degree of freedom. As the first degree of freedom varies periodically and rapidly, we perform a Poincaré section of the plane $\left(x_{3}, y_{3}\right)$ with the condition $y_{1}=0$ as section. Figure 9 shows such a Poincaré section. In this case, as expected, the orbit is elliptic without any oscillations due to the first proper frequency. In order to highlight the smoothness of the curve, we superpose the complete motion on the section in a zoom of the plot, presented in Fig. 10. It is obvious that the first degree of freedom introduces small, rapid, periodic deformations on the regular motion of the second degree of freedom but modifies neither the shape of curves nor the dynamical behavior.

\section{Conclusion}

We have studied the global, coupled resonant dynamics of Mercury and calculated the amplitude of resonance areas. We found that the resonance area of the actual position of Mercury is around 14 degrees in ecliptic obliquity. We also studied the interaction among the two degrees of freedom used to describe the rotation of Mercury. The associated proper periods are very distinct and the analysis of the mutual perturbation require different tools: (i) the impact of the slow variable on the fast one is studied through the adiabatic invariant, whereas (ii) the impact of the fast variable on the slow one can be described 
(a)

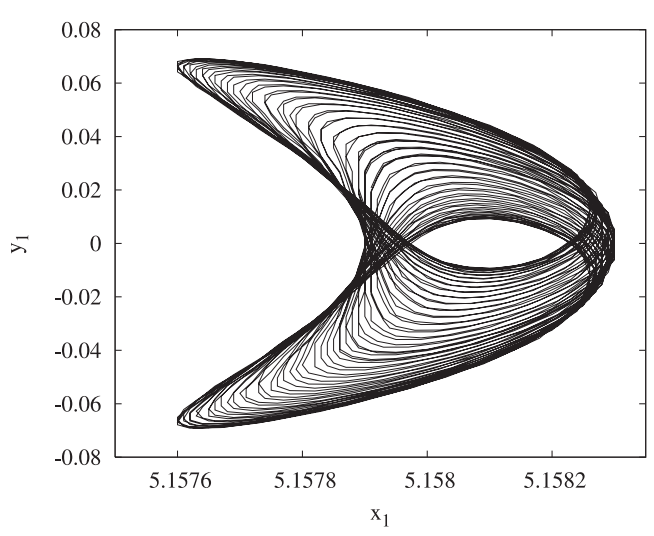

(b)

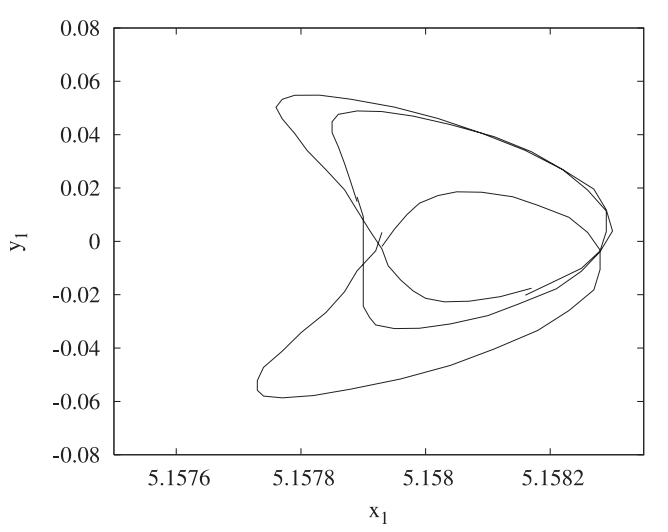

Fig. 8. The orbit in the plane $\left(x_{1}, y_{1}\right)$ in the case of the Hamiltonian $\mathcal{K}_{4}$. Panel a) shows the orbit over 1070 years. Panel b) shows parts of the orbit taken at three different times and plotted over 16 years in each case.

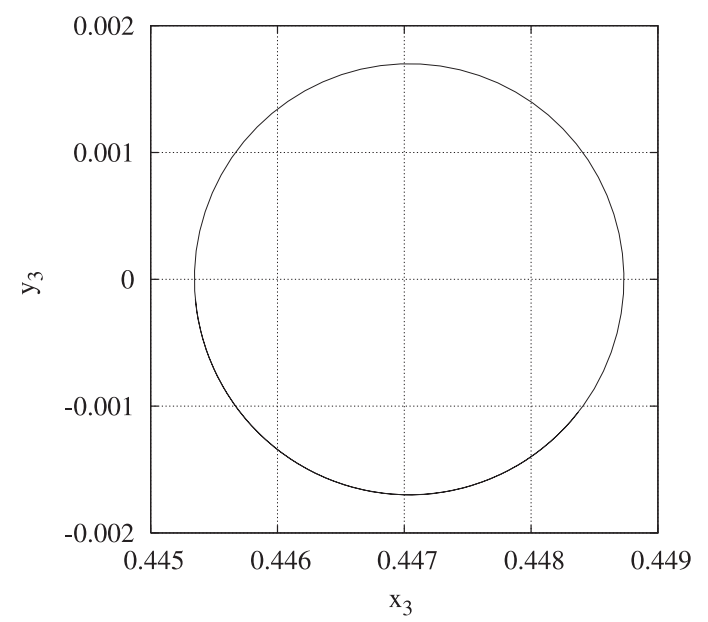

Fig. 9. Cross section of the plane $\left(x_{3}, y_{3}\right)$ for $y_{1}=0$. The motion is characterized by a pendulum-like section.

through Poincaré sections. In addition, using the adiabatic invariant, we establish a simple analytical model of the coupled

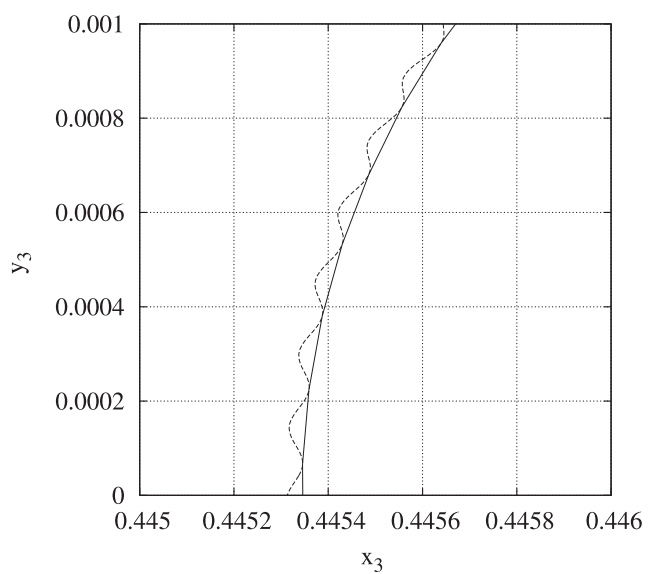

Fig. 10. The complete curve (dashed lines) and the section (black line). The cross section is the smooth curve and presents no oscillations as expected.

rotation of Mercury providing a right qualitative description of motion. This analytical model can be used for various applications to analyze observations, as well as to understand dynamical phenomena such as the capture in resonance. For example, the influence of the precession-rotation of Mercury on the orbital plane of an artificial satellite can be studied easily. These studies will be very useful for analyzing observations of Hermean probes such as MESSENGER or BepiColombo.

Acknowledgements. We wish to thank Professor J. Henrard for many enlightening discussions and suggestions. Nicolas Rambaux acknowledges the support of a Prodex/ESA contract C90253 at the FUNDP.

\section{References}

Anderson, J. D., Colombo, G., Esposito, P. B., Lau, E. L., \& Trager, G. B. 1987, Icarus, 71,337

Beletskii, V. V. 1972, Celest. Mech., 6, 356

Bouquillon, S., Kinoshita, H., \& Souchay, S. 2003, Celest. Mech. Dyn. Astron., 86,29

Celletti, A., \& Falcolini, C. 1992, Celest. Mech. Dyn. Astron., 53, 113

Celletti, A., \& Chiercia, L. 2000, Celest. Mech. Dyn. Astron., 76, 229

Colombo, G. 1965, Nature, 208, 575

Deprit, A. 1967, Am. J. Phys., 35, 424

D'Hoedt, S., \& Lemaitre, A. 2004a, Celest. Mech. Dyn. Astron., 89, 267

D'Hoedt, S., \& Lemaitre, A. 2004b, in Transit of Venus: New Views of the Solar System and Galaxy, ed. D. W. Kurtz, \& G. E. Bromage, Proc. IAU Coll., 16 , under press

D'Hoedt, S., Lemaitre, A., \& Rambaux, N. 2006, Celest. Mech. Dyn. Astron., in press

Froeschlé, C. 1972, A\&A, 16, 172

Henrard, J. 2005, in: Hamiltonian Dynamics: theory and applications ed. Giorgilli (Springer)

Lemaitre, A., D’Hoedt, S., \& Rambaux, N. 2006, Celest. Mech. Dyn. Astron., in press

Peale, S. J. 1969, AJ, 74, 483

Peale, S. J. 1974, AJ, 79, 72

Pettengill, G. H., \& Dyce, R. B. 1965, Nature, 206, 1240

Rambaux, N., \& Bois, E. 2004, A\&A, 413, 381 